\title{
STM study of site selective hydrogen adsorption on $\operatorname{Si}(111) 7 \times 7$
}

\author{
Ivy Razado-Colambo, Hanmin M. Zhang, Roger I. G. Uhrberg and Göran V. Hansson
}

\section{Linköping University Post Print}

\section{Tweet}

N.B.: When citing this work, cite the original article.

Original Publication:

Ivy Razado-Colambo, Hanmin M. Zhang, Roger I. G. Uhrberg and Göran V. Hansson, STM study of site selective hydrogen adsorption on $\mathrm{Si}(111) 7 \times 7,2005$, Physical Review B. Condensed Matter and Materials Physics, (71), 23, 235411.

http://dx.doi.org/10.1103/PhysRevB.71.235411

Copyright: American Physical Society http://www.aps.org/

Postprint available at: Linköping University Electronic Press

http://urn.kb.se/resolve?urn=urn:nbn:se:liu:diva-17366 


\title{
STM study of site selective hydrogen adsorption on $\mathrm{Si}(111) 7 \times 7$
}

\author{
I. C. Razado, H. M. Zhang, R. I. G. Uhrberg, and G. V. Hansson \\ Department of Physics and Measurement Technology, Linköping University, S-581 83 Linköping, Sweden \\ (Received 2 December 2004; revised manuscript received 17 February 2005; published 16 June 2005)
}

\begin{abstract}
Adsorption of atomic hydrogen has been studied by scanning tunneling microscopy (STM) and photoelectron spectroscopy with a focus on the different adsorption sites provided by the $\mathrm{Si}(111) 7 \times 7$ surface. At low temperature, the hydrogen atoms adsorb preferentially on adatoms while at elevated temperatures the rest atoms are the first to become hydrogen terminated. The hydrogen-terminated rest atoms are no longer visible in the STM images and the surrounding adatoms appear brighter compared to the clean $7 \times 7$ surface. This indicates that there is a local charge transfer back to the adatoms from the rest atoms. Three kinds of modified triangular subunit cells of the $7 \times 7$ reconstruction have been identified corresponding to one, two, and three hydrogen-terminated rest atoms, respectively. A detailed study of the apparent height using STM line profiles through the adatom and rest atom positions on the surface is presented. These line profiles show a characteristic and reproducible variation of the apparent heights of the adatoms for the different kinds of triangular subunit cells and the changes are interpreted in terms of charge transfer. The very local nature of the charge transfer is concluded from the fact that only the hydrogen termination of neighboring rest atoms is significantly affecting the apparent height of an adatom.
\end{abstract}

DOI: $10.1103 /$ PhysRevB.71.235411

PACS number(s): 68.35. $-\mathrm{p}, 73.20 .-\mathrm{r}, 79.60 .-\mathrm{i}$

\section{INTRODUCTION}

Studies of hydrogen adsorption on silicon surfaces have been of great interest in surface science. Adsorption of hydrogen on silicon changes the properties of the surface since it can passivate the dangling bonds. ${ }^{1}$ Over the years there have been several investigations of this type on the $\mathrm{Si}(111)$ $7 \times 7$ surface but they have mainly dealt with larger exposures resulting in $7 \times 1$ and $1 \times 1$ surfaces. ${ }^{2-6}$ Most of the studies have focused on adsorption where the adatoms are replaced by hydrogen atoms. The scanning tunneling microscopy (STM) studies by Mortensen et al. ${ }^{7}$ and Boland ${ }^{8}$ of the room-temperature exposure of the $\mathrm{Si}(111) 7 \times 7$ surface showed that the adatom layer can be rearranged revealing the layer below the adatoms. In the STM study done by Rogers and Tiedje, ${ }^{9}$ the equilibrium distribution of chemisorbed hydrogen on the different adatoms on the $\operatorname{Si}(111) 7 \times 7$ surface has been determined by counting hydrogen-terminated adatoms in STM images of exposed surfaces.

Our study is different from the previous investigations in the sense that we focus on site-specific adsorption on the rest atom sites of the $7 \times 7$ surface. The exposures were done in such a way that the $7 \times 7$ atomic structure was kept and only electronic charge transfer occurred. In the paper by Avouris and Wolkow, ${ }^{10}$ it has been reported that the activation barrier for reaction at adatom sites is larger than that for reaction at rest atom sites, leading to a faster reaction rate at rest atom sites at elevated temperatures, in agreement with our STM observations. In the work by Lo et al., ${ }^{1}$ adsorption and desorption of single hydrogen atoms on the $7 \times 7$ surface at elevated temperatures have been studied by STM. It was observed that hydrogen atoms adsorb preferentially on top of rest atoms. They studied the diffusion path of single hydrogen atoms on the $\mathrm{Si}(111) 7 \times 7$ surface with a variabletemperature scanning tunneling microscope. Based on density functional calculations, Vittadini and Selloni earlier had proposed that the most stable adsorption site for $\mathrm{H}$ atoms on $\mathrm{Si}(111)$ is the rest atom site. ${ }^{11}$
The $\operatorname{Si}(111) 7 \times 7$ surface was characterized by Takayanagi et al. ${ }^{12}$ in 1985 as a dimer-adatom-stacking (DAS) fault structure shown in Fig. 1. The unit cell consists of two halves: the faulted and the unfaulted triangular subunit cells. The first layer of the unit cell is made up of 12 adatoms which can be classified as either corner or center adatoms. A corner adatom has one rest atom neighbor while a center adatom has 2 rest atom neighbors. The second layer which is about $1 \AA$ below the first layer contains 6 rest atoms. Each adatom and rest atom has one dangling bond. The cornerhole atoms are $4.4 \AA$ below the adatom layer, having one dangling bond each. In total, the reconstructed $7 \times 7$ unit cell has 19 dangling bonds as compared to the 49 dangling bonds that the unreconstructed $1 \times 1$ unit cell has in the same area. In STM, these dangling bonds are the primary source of the

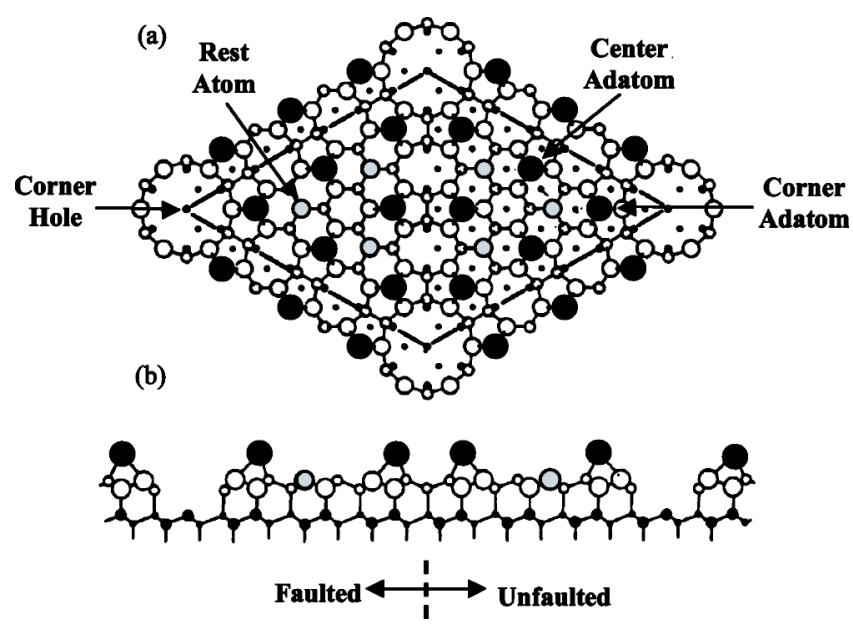

FIG. 1. DAS model of the $7 \times 7$ structure proposed by Takayanagi et al. (Ref. 12). (a) Top view. The solid circles are the adatoms and the shaded circles are the rest atoms. (b) A cross-sectional side view through the mirror plane of the unit cell. 
tunneling current. On the clean $7 \times 7$ surface, the 12 adatoms, 6 rest atoms, and 1 corner hole atom together contribute 19 electrons to the dangling bond surface states. From a theoretical study of the electronic structure of the $7 \times 7$ surface, ${ }^{13}$ it was concluded that there is a transfer of electrons from the adatoms to the rest atoms and to the cornerhole atom which results in doubly occupied dangling bond states on these atoms. In an empty-state STM image, only the adatoms are observed, while in a filled-state STM image, both the adatoms and the rest atoms are visible.

The aim of this work is to study the charge transfer induced by hydrogen exposure of the $\operatorname{Si}(111) 7 \times 7$ surface. In particular, we have studied the changes when rest atom dangling bonds are passivated by hydrogen, leading to the electron back transfer to the surrounding adatoms. The local structure of the triangular subunit cells of the $\mathrm{Si}(111) 7 \times 7$ surface after low hydrogen exposures has not been previously studied by scanning tunneling microscopy. By using the powerful capabilities of STM, the surface could be probed on an atomic scale. In this study we show that at an elevated temperature $\left(\sim 340{ }^{\circ} \mathrm{C}\right)$, hydrogen atoms adsorb preferentially on top of rest atoms, inducing a local rest atom to adatom charge transfer. The mere fact that it is always the three adatoms surrounding a specific rest atom that are affected confirms, by symmetry, the adsorption on rest atom sites as was also alluded to in Ref. 1. The preferential adsorption on rest atom sites is also verified by valence band photoelectron spectroscopy.

\section{EXPERIMENTAL DETAILS}

The experiments were carried out in a homebuilt UHV STM molecular beam epitaxy (MBE) chamber with base pressure below $5 \times 10^{-11}$ Torr. It is equipped with lowenergy electron diffraction (LEED) and quadrupole mass spectroscopy (QMS). The Si(111) samples, cut from an Sbdoped silicon wafer, were cleaned using the Ishizaka-Shiraki method. ${ }^{14}$ Each sample was loaded into the preparation chamber using a load-lock system and outgassing was done for several hours by resistively heating the sample. The thin oxide layer was removed by heating the sample to $\sim 900{ }^{\circ} \mathrm{C}$ and high temperature flashing up to $1150^{\circ} \mathrm{C}$ was employed, maintaining a pressure below $5 \times 10^{-10}$ Torr, in order to obtain a clean and defect-free $\mathrm{Si}(111) 7 \times 7$ surface. The hydrogen gas quality was checked using the mass spectrometer. Molecular hydrogen was introduced through a leak valve into the preparation chamber. A tungsten filament was used to dissociate the molecular hydrogen into atomic hydrogen. The filament was placed $7 \mathrm{~cm}$ above the sample surface and was heated to $\sim 1800{ }^{\circ} \mathrm{C}$ during exposures in the range of 0-10 langmuir ( $1 \mathrm{~L}=10^{-6}$ Torr s) of molecular hydrogen. The sample temperature was varied between 290 and $380{ }^{\circ} \mathrm{C}$. After hydrogen exposure, the samples were transferred to the STM chamber. All images were recorded at room temperature in constant-current mode with a Pt/Ir tip. Temperatures were measured using an IR pyrometer. Photoemission spectra were obtained at BL I311 at MAX-lab using a Scienta analyzer with a $15^{\circ}$ acceptance angle. The hydrogen exposures in the photoemission and STM studies were done in a similar way.
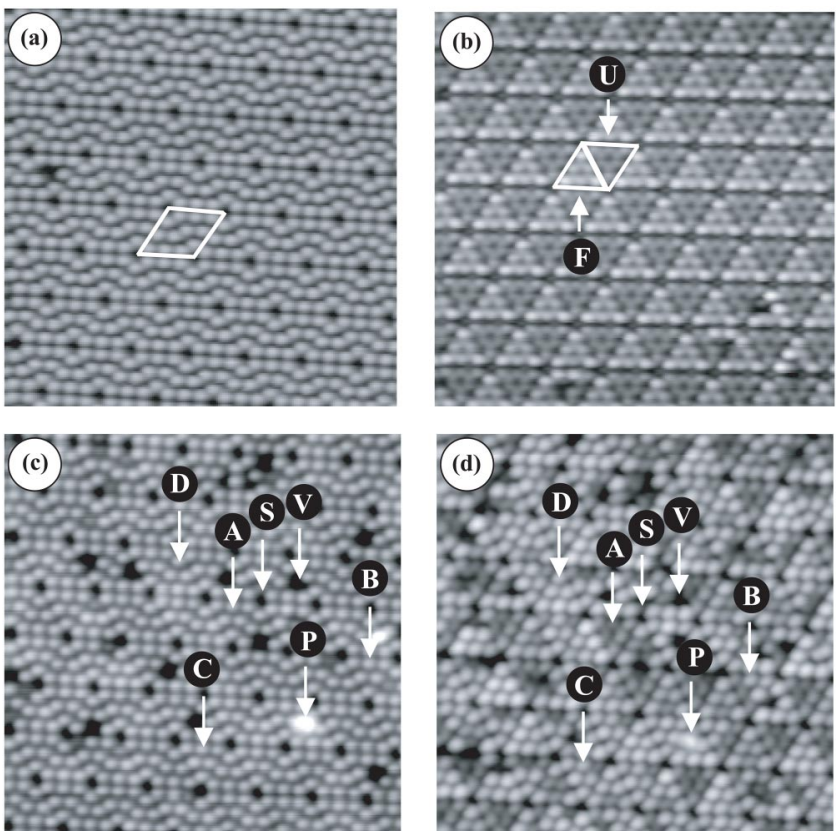

FIG. 2. STM overviews comparing the clean $7 \times 7$ and the hydrogen-exposed surface (both filled- and empty-state images). (a) Empty-state STM image of the $\mathrm{Si}(111) 7 \times 7$ surface recorded with a tip voltage of -2 V. (b) Filled-state STM image of the Si(111) $7 \times 7$ surface recorded with a tip voltage of $+1.4 \mathrm{~V}$. (c) and (d) Empty- and filled-state STM images of the hydrogen-exposed $\mathrm{Si}(111) 7 \times 7$ surface recorded at a tip voltage of $-2 \mathrm{~V}$ and $+2 \mathrm{~V}$, respectively. The size of all images is $180 \AA \times 180 \AA$. The tunneling current was $0.3 \mathrm{nA}$.

\section{RESULTS AND DISCUSSION}

Vittadini and Selloni ${ }^{11}$ proposed, based on density functional calculations, that the most stable adsorption site for $\mathrm{H}$ atoms on $\mathrm{Si}(111)$ is the rest atom site. Adsorption of hydrogen on an adatom is a metastable state characterized by a shorter lifetime compared to the rest atom site. In the work of Lo et al., ${ }^{1}$ they estimated the difference in the binding energy to be $\sim 0.2 \mathrm{eV}$. We have optimized the hydrogen exposure and temperature to promote the rest atom adsorption and we find that more than $70 \%$ of the rest atoms can be hydrogen terminated before any significant vacancy formation starts. Under these conditions, less than $4 \%$ of the adatoms are hydrogen terminated. Since the surface retains the $7 \times 7$ reconstruction, it is a very suitable model system for a study of the hydrogen-induced charge redistribution between different types of surface atoms.

Figure 2 shows a comparison of STM images of the clean $\operatorname{Si}(111) 7 \times 7$ surface and the hydrogen-exposed surface. Figure $2(\mathrm{a})$ is an STM image of the clean $\mathrm{Si}(111) 7 \times 7$ surface obtained with a tip bias of $-2 \mathrm{~V}$ which reflects the spatial distribution of the unoccupied surface electronic states. The dominant features in the image are the adatoms and corner holes of the $7 \times 7$ reconstruction. The $7 \times 7$ unit cell is outlined and the 12 adatoms are clearly seen. In images of the unoccupied states, the two kinds of triangular halves of the $7 \times 7$ unit cell appear indistinguishable. There is only one adatom vacancy seen in the STM image which means that 


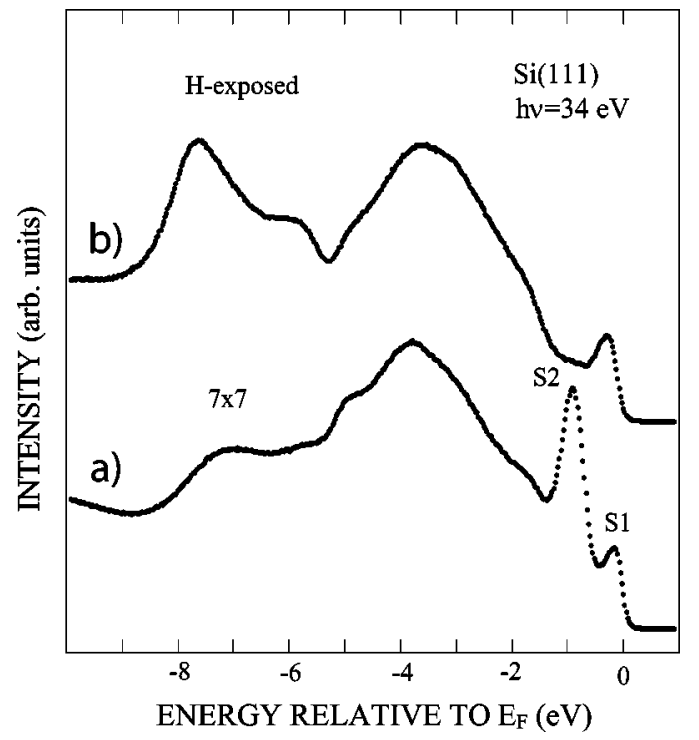

FIG. 3. Valence band spectra obtained from the clean $\operatorname{Si}(111)$ $7 \times 7$ surface (a) and after $\mathrm{H}$ exposure (b). The photon energy was $34 \mathrm{eV}$ and the spectra were recorded in normal emission with a $15^{\circ}$ acceptance angle. The $\mathrm{H}$-exposed surface shows a strong reduction of the rest atom emission $(S 2)$ while the adatom peak $(S 1)$ increases slightly compared to the $7 \times 7$ surface.

the defect density is very low $(<0.2 \%$ of the total number of adatoms). Figure 2(b) shows a filled-state STM image of the $7 \times 7$ surface obtained with a tip bias of $+1.4 \mathrm{~V}$. The 12 adatoms and the difference between the two halves are clearly seen. The faulted half $(F)$ appears higher as compared to the unfaulted half $(U)$ and corner adatoms appear higher than the center adatoms. The rest atoms between the adatoms are also visible. This occurs because the states associated with the doubly occupied dangling bonds of the rest atoms contribute to the tunneling current when probing the filled states while they are not visible when probing the empty states.

Figure 2(c) shows an empty-state STM image of the hydrogen-exposed surface obtained with a tip bias of $-2 \mathrm{~V}$. The surface appears almost the same as the empty-state STM image of the clean surface aside from the increased number of vacancies $(V)(1 \%)$ and some bright protrusions $(P)$. The appearance of the filled-state STM image of the same area in Fig. 2(d), obtained with a tip bias of $+2 \mathrm{~V}$, is more interesting. Most of the adatoms are brighter while most of the rest atoms are no longer visible. This suggests that at $\sim 340{ }^{\circ} \mathrm{C}$ most of the rest atom dangling bonds were saturated by atomic hydrogen, which is in agreement with the theoretical calculation of site selective adsorption on rest atoms. ${ }^{11}$

The site selectiveness is also confirmed by photoemission. Figure 3(a) shows a valence band spectrum from the clean $\mathrm{Si}(111) 7 \times 7$ surface. The two peaks closest to the Fermi level correspond to emission from the adatom $(S 1)$ and the rest atom $(S 2)$ states, respectively. These assignments are firmly based on theoretical investigations ${ }^{13}$ and a currentimaging-tunneling-spectroscopy (CITS) study. ${ }^{15}$ In the CITS images the adatoms appeared at a sample bias of $-0.35 \mathrm{eV}$ and the rest atoms at $-0.85 \mathrm{eV}$, which agree very well with the energies of $S 1$ and $S 2$. After a hydrogen exposure similar to that of Figs. 2(c) and 2(d), the emission from the rest atom states is dramatically reduced as shown by Fig. 3(b). This is evidence for hydrogen adsorption on the rest atoms since the electrons of the $\mathrm{Si}-\mathrm{H}$ bond have energies around $-5 \mathrm{eV},{ }^{16}$ which explains the reduced intensity at the position of $S 2$. The emission from the adatom states, on the other hand, is slightly increased as expected due to back transfer of the charge to the adatoms. The increased number of electrons in the $S 1$ state results in a shift away from the Fermi level. These observations confirm the picture that the hydrogen adsorbs preferentially at the rest atom sites. The intensities of the $S 1$ and $S 2$ peaks can be obtained by curve fitting. The $S 2$ contribution in the spectrum from the hydrogen-exposed surface is just $15 \%$ of that of the clean surface. This is in accordance with the observation that more than $70 \%$ of the rest atoms are hydrogen terminated in Fig. 2(d) at the same time as a very small fraction of the adatoms are hydrogen terminated.

We will consider four types of triangular subunit cells on the hydrogen-exposed surface which are labeled as $A, B, C$, and $D$ in Fig. 2(d). $A$ is a triangular subunit cell without any adsorbed hydrogen atom while $B, C$, and $D$ have one, two, and three hydrogen-terminated rest atoms, respectively. These triangles have characteristic apparent heights of the different adatoms resulting from a local charge transfer back to the adatoms from the rest atoms. However, in the emptystate image, it is not possible to observe any effect on the adatoms. The defect labeled $V$ appears as a deep depression also when probing the filled states of the sample which means that it corresponds to a vacancy in the adatom layer, created by hydrogen-induced breaking of the adatom backbonds. The adatoms thus removed stay on the surface and result in features like the one labeled $P$. A few of the adatoms also appear darker than usual both in the empty-state and filled-state STM images such as the one labeled $S$. This suggests that the dangling bond states associated with those adatoms have been passivated by a hydrogen atom.

The reduction in the number of rest atoms observed when imaging the filled states suggests that most of the dangling bonds on the rest atoms in the $7 \times 7$ structure are saturated with hydrogen atoms after exposure. This is hard to observe in the overview in Fig. 2(d) but it will be described in detail below. In this study, the rest atoms are clearly more reactive than the adatoms. This is in agreement with other studies that hydrogen atoms preferentially adsorb on top of rest atoms at elevated temperatures. ${ }^{1}$

The triangular subunit cells used for the detailed charge transfer study have been chosen such that they do not contain hydrogen-terminated adatoms. The direction of the line scans for the faulted and unfaulted halves of the triangular subunit cells are shown in Fig. 4.

Figures 5(a)-5(h) are filled-state STM images of the different triangular subunit cells with their corresponding line profiles $i, i i$, and iii passing through the adatoms and $i v, v$, and vi passing through both the adatoms and rest atoms. All images were recorded with a $+2-\mathrm{V}$ tip voltage and a 0.3-nA tunneling current. A positive tip bias was used in order to probe the occupied states of the surface and to image both the adatoms and rest atoms of the faulted and unfaulted 
(a)

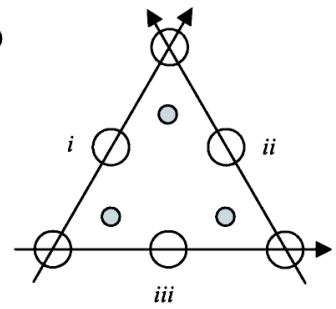

(c)

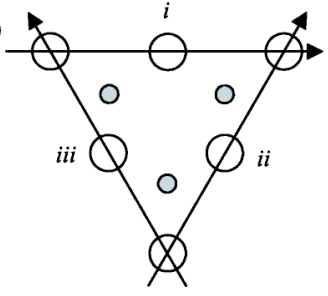

(b)
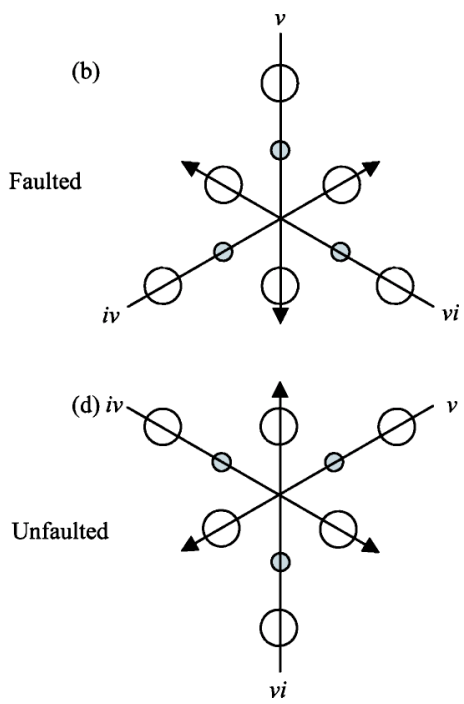

FIG. 4. (a) and (c) show the directions of the line scans through adatom positions, while (b) and (d) show line scan directions through both adatom and rest atom positions.

halves. A least-mean-squares-fitted plane was subtracted to produce flat images.

Figures 5(a) and 5(b) were obtained from clean $\mathrm{Si}(111)$ $7 \times 7$ showing the faulted and unfaulted halves as indicated by the white arrows, respectively. The faulted half appears brighter as compared to the unfaulted half. Comparing the corner adatoms of the two halves we find an apparent height difference of $0.3 \AA$. Moreover, the corner adatoms appear brighter than the center adatoms. In the faulted half, the height difference between the center adatoms and the corner adatoms, which is around $0.5 \AA$, is clearly seen in the line profiles. In the unfaulted half, the height difference between the two kinds of adatoms is smaller $(0.2 \AA)$. On the clean surface, an electronic charge transfer takes place from the adatoms to the rest atoms, such that the corresponding dangling bonds of the rest atoms are fully occupied. ${ }^{13}$

Figure 5(c) shows a faulted triangular subunit cell with one hydrogen-terminated rest atom $(1 \mathrm{H})$. Comparing it with the clean surface, one rest atom is no longer visible in the triangular subunit cell as seen in scan vi. This is because the dangling bond state of the rest atom was passivated by the strong $\mathrm{Si}$ and $\mathrm{H}$ covalent bond. The small bump between the two corner adatoms does not correspond to a rest atom position, which is verified by superimposing the line profile $5(\mathrm{a}), v i$, of the clean surface. The small bump in the dashed curve is due to the finite size of the tip which means that the tip cannot go as deep between the two center adatoms as it can at the hydrogen-terminated rest atom position.

The three adatoms surrounding the hydrogen-terminated rest atom-two center adatoms and one corner adatomappeared higher. This is caused by a charge transfer from the rest atom back to the adatoms. From the line profile, the apparent height of the corner adatom was increased by about $0.4 \AA$ while for the center adatoms it was increased by about $0.9 \AA$. The other adatoms in the subunit cell retained their original apparent heights. Figure 5(d) shows an unfaulted triangular subunit cell with one hydrogen-terminated rest atom. The adatoms surrounding the hydrogen-passivated rest atom became brighter than usual also in this case. The apparent height difference between the corner adatom on the clean surface and the corner adatom near the hydrogenterminated rest atom is $0.8 \AA$ while for the center adatoms it is $0.6 \AA$.

Figure 5(e) shows a faulted triangular subunit cell with two hydrogen-terminated rest atoms $(2 \mathrm{H})$. It shows that the five neighboring adatoms became brighter than before; in particular, the center adatom neighboring both hydrogenterminated rest atoms has the largest apparent height increase. Specifically, the apparent height difference as compared to the center adatom on the clean surface is about $1.1 \AA$. The apparent height change for the other two center adatoms is $0.8 \AA$ while the two corner adatoms have an apparent height change of $0.4 \AA$. Figure $5(\mathrm{f})$ is an unfaulted triangular subunit cell with two hydrogen-terminated rest atoms as well. The effect is similar to that in the faulted half. The five adatoms neighboring the hydrogen-terminated rest atoms increased their apparent heights. The center adatom with two neighboring hydrogen-terminated rest atoms has the largest change in apparent height which is $1 \AA$. The two other center adatoms have an apparent height change of $0.7 \AA$ while the corner adatoms have changed by $0.5 \AA$ in the apparent height.

Figure $5(\mathrm{~g})$ shows a faulted triangular subunit cell where all three rest atoms are saturated with hydrogen $(3 \mathrm{H})$. All the adatoms increased their apparent heights as seen in the line profiles. The average apparent height difference of the corner adatoms as compared to the clean surface is $0.4 \AA$ while for the center adatoms it is $0.9 \AA$. Figure $5(\mathrm{~h})$ shows an unfaulted half cell with all three rest atoms being hydrogen terminated. The average apparent height difference for the corner adatoms as compared with the clean surface is $0.5 \AA$ while for the center adatoms it is $0.9 \AA$. All center adatoms have two neighboring hydrogen-terminated rest atoms, so the charge redistribution is the same for all three center adatoms.

Table I shows the average change in apparent heights for the four kinds of adatoms in the $7 \times 7$ unit cell that are affected by hydrogen termination of the neighboring rest atoms: the corner adatoms in the faulted half (I), the center adatoms in the faulted half (II), the corner adatoms in the unfaulted half (III), and the center adatoms in the unfaulted half (IV). Several triangular subunit cells for each type $(1 \mathrm{H}$, $2 \mathrm{H}, 3 \mathrm{H})$ were investigated in order to obtain the average values for the apparent height changes. The uncertainties given correspond to the standard deviation. The change in the apparent height of the corner adatoms (I and III) is expected to be independent of the number of hydrogenterminated rest atoms since the corner adatoms have just one neighboring rest atom. The data are consistent with this idea and the values are, for the faulted half, about $0.4 \AA$ and about $0.6 \AA$ for the unfaulted half. There are two types of center adatoms, one with one neighboring hydrogenterminated rest atom as in the cases of $1 \mathrm{H}$ and $2 \mathrm{H}$ and one with two neighboring hydrogen-terminated rest atoms as in the cases of $2 \mathrm{H}^{\prime}$ and $3 \mathrm{H}$. More charge is transferred to the center adatom that neighbors two rest atoms passivated with hydrogen as compared to the center adatom that has only one hydrogen-terminated rest atom neighbor as seen in Table I. 

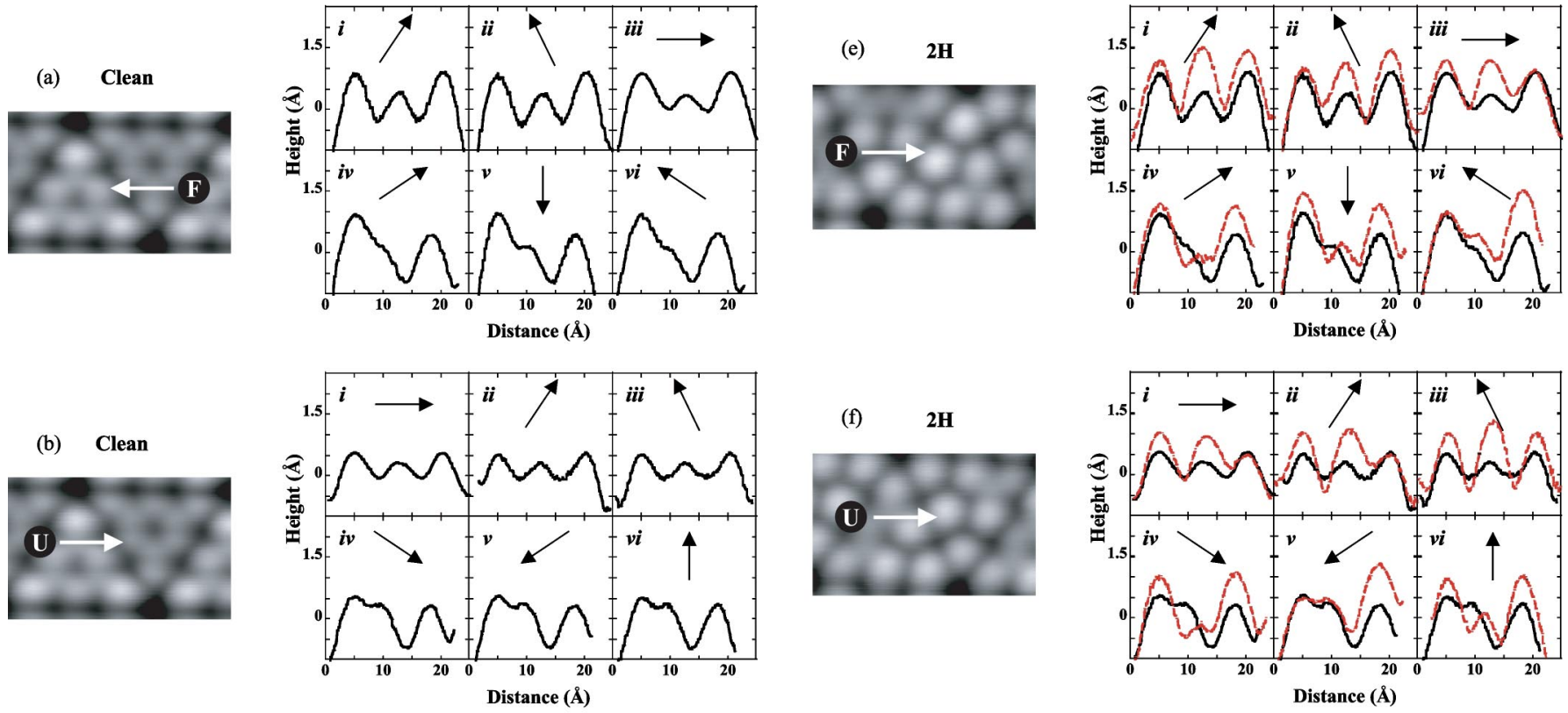

(c) $\quad \mathbf{1 H}$
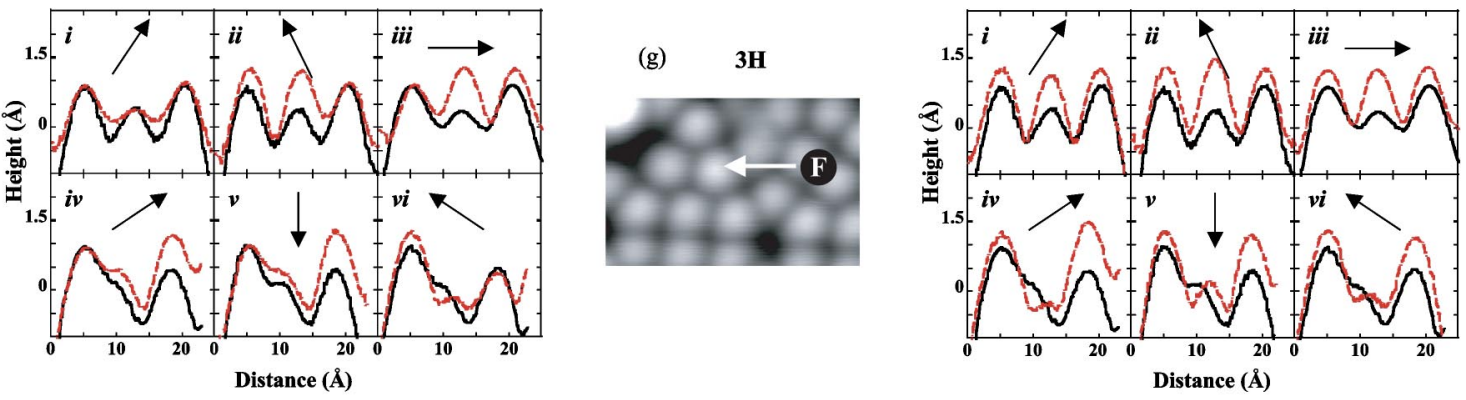

(d)
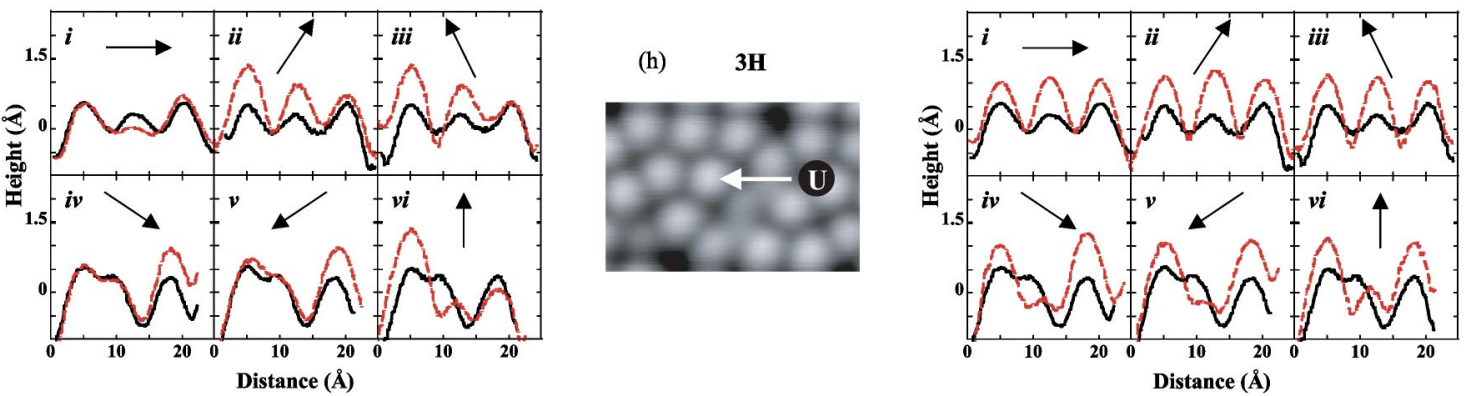

FIG. 5. (Color online) Filled-state STM images of the triangular subunit cells together with line profiles through the adatoms and rest atoms. All images were taken with a $+2-\mathrm{V}$ tip bias and a constant tunneling current of $0.3 \mathrm{nA}$. Image size is $23 \AA \times 33 \AA$. The directions of the line scans are illustrated in Fig. 4. The solid lines represent the line scans for the clean unit cells and the dashed lines represent the line scans for the hydrogen-exposed unit cells. The superposition of the two lines clearly shows the increase in apparent heights of the adatoms when a neighboring rest atom becomes hydrogen terminated. The line scans passing through both the adatoms and rest atoms $(i v, v, v i)$ show the disappearance of the rest atom peak when it is hydrogen terminated.

Figure 5(a), iv, $v$, and $v i$, shows line profiles passing through the rest atom and adatom positions in the faulted half cell of the clean $7 \times 7$ surface. The shoulder near the corner adatom shows the rest atom position that is situated $4.4 \AA$ from the corner adatom. There is a more pronounced height difference between the adatom and the rest atom in the faulted side as compared with the unfaulted side. When the rest atom is hydrogen terminated, no peak appears at the rest atom position. Figure 5(c), vi, shows that one rest atom is hydrogen terminated since no shoulder appears near the corner adatom in the dotted curve. The small bump in the dashed curve is an artifact due to the finite size of the tip as mentioned previously. The increase in apparent height of the neighboring adatoms is also evident in these line profiles. In Fig. 5(d), vi, the same effect is observed since the shoulder near the corner adatom disappeared as clarified by the superposition of line profile 5(b), vi. However, the increase in apparent height of the corner adatom near the reacted rest atom is higher by $0.5 \AA$ in the unfaulted half as compared to the faulted half.

Figure 5(e), iv and $v$, shows that two rest atoms are saturated with hydrogen as manifested by the absence of shoul- 
TABLE I. Changes in apparent heights $(\Delta h)$ of the corner adatoms (I) and center adatoms (II) in the faulted half and corner adatoms (III) and center adatoms (IV) in the unfaulted half. The $1 \mathrm{H}$ and $2 \mathrm{H}$ columns correspond to adatoms with one neighboring hydrogen-terminated rest atom and $2 \mathrm{H}^{\prime}$ and $3 \mathrm{H}$ correspond to adatoms with two neighboring hydrogen-terminated rest atoms.

\begin{tabular}{|c|c|c|c|c|}
\hline \multirow[b]{2}{*}{ Adatom } & \multicolumn{4}{|c|}{$\Delta h(\AA)$} \\
\hline & $1 \mathrm{H}$ & $2 \mathrm{H}$ & $2 \mathrm{H}^{\prime}$ & $3 \mathrm{H}$ \\
\hline I & $0.43 \pm 0.04$ & $0.30 \pm 0.08$ & & $0.43 \pm 0.08$ \\
\hline II & $0.79 \pm 0.08$ & $0.68 \pm 0.07$ & $0.98 \pm 0.07$ & $1.00 \pm 0.09$ \\
\hline III & $0.62 \pm 0.14$ & $0.61 \pm 0.13$ & & $0.64 \pm 0.07$ \\
\hline IV & $0.74 \pm 0.11$ & $0.77 \pm 0.16$ & $1.10 \pm 0.16$ & $1.10 \pm 0.17$ \\
\hline
\end{tabular}

ders near the corner adatom. It is also evident from the two line profiles that the increase in apparent height on the two center atoms is higher as compared to the two corner adatoms and we can speculate that more charge is transferred back from the rest atoms to the center adatoms than to the corner adatoms. In particular the center adatom, which has two hydrogen-terminated rest atom neighbors, has a very large apparent height increase $(\sim 1.0 \AA)$ as seen in line profiles of Fig. 5(e), $i$ and vi. Very similar effects are observed on the unfaulted half in Fig. 5(f), $i v, v$, and vi. In Fig. 5(g), $i v$, $v$, and $v i$, we can see that all rest atom dangling bonds are passivated by hydrogen atoms in the faulted half. The change in apparent height is again larger for the center adatoms as compared to the corner adatoms. This is also illustrated in the unfaulted half but less pronounced than in the faulted half.

These observations suggest that the amount of reverse electronic charge transfer from the rest atoms back to the adatoms depends on the type of adatom. There is a strong connection to the original charge distribution and apparent height of the atoms on the $\operatorname{Si}(111) 7 \times 7$ surface. In general, the changes in the apparent height are larger for adatoms that appear low on the clean surface. As a result, the apparent heights of all atoms are quite uniform in $7 \times 7$ unit cells where all six rest atoms are hydrogen terminated. A possible explanation for this behavior is the fact that the number of dangling bond electrons on the adatoms increases from 5 to 12 when all rest atoms and the corner-hole atoms become hydrogen terminated. When probing the occupied states, a small variation of the low electron concentration will have a large impact on images of the $7 \times 7$ surface. On the hydrogen-exposed surface the effect of such a variation will be much smaller.

\section{SUMMARY}

The local changes when hydrogen is adsorbed on $\mathrm{Si}(111)$ $7 \times 7$ were studied using filled-state STM images. It was observed that hydrogen atoms preferentially adsorb on top of rest atoms at elevated temperatures $\left(\sim 340{ }^{\circ} \mathrm{C}\right)$. In the filledstate STM images and the line profiles, the reacted rest atoms can no longer be seen and the adatoms neighboring the hydrogen-saturated rest atoms became brighter, suggesting that there is a reverse charge transfer from the rest atoms back to the surrounding adatoms. This was manifested by an increase in apparent heights of the adatoms neighboring reacted rest atom site(s). The charge transfer to the adatoms is influenced by the number of neighboring hydrogenterminated rest atoms. The center adatom with two hydrogen-terminated rest atom neigbors gets most of the charge. By analyzing the change in apparent height of many adatoms one can conclude that the charge redistribution is very local. There is no evidence of a charge redistribution that extends to second-nearest-neighboring adatoms when a rest atom becomes hydrogen terminated.

\section{ACKNOWLEDGMENT}

This work was supported by the Swedish Research Council
${ }^{1}$ R. L. Lo, I. S. Hwang, M. S. Ho, and T. T. Tsong, Phys. Rev. Lett. 80, 5584 (1998).

${ }^{2}$ J. Z. Que, M. W. Radny, and P. V. Smith, J. Phys.: Condens. Matter 8, 4205 (1996).

${ }^{3}$ F. Owman and P. Mårtensson, Surf. Sci. Lett. 303, L367 (1994).

${ }^{4}$ F. Owman and P. Mårtensson, Surf. Sci. 324, 211 (1995).

${ }^{5}$ D. Rogers and T. Tiedje, J. Vac. Sci. Technol. B 15, 1641 (1997).

${ }^{6}$ A. Kraus, M. Hanbücken, T. Koshikawa, and H. Neddermeyer, Appl. Surf. Sci. 177, 292 (2001).

${ }^{7}$ K. Mortensen, D. M. Chen, P. J. Bedrossian, J. A. Golovchenko, and F. Besenbacher, Phys. Rev. B 43, 1816 (1991).

${ }^{8}$ J. J. Boland, Surf. Sci. 244, 1 (1991).
${ }^{9}$ D. Rogers and T. Tiedje, Phys. Rev. B 53, R13227 (1996).

${ }^{10} \mathrm{Ph}$. Avouris and R. Wolkow, Phys. Rev. B 39, 5091 (1989).

${ }^{11}$ A. Vittadini and A. Selloni, Phys. Rev. Lett. 75, 4756 (1995).

${ }^{12}$ K. Takayanagi, Y. Tanishiro, M. Takahashi, and S. Takahashi, J. Vac. Sci. Technol. A 3, 1502 (1985).

${ }^{13}$ J. E. Northrup, Phys. Rev. Lett. 57, 154 (1986).

${ }^{14}$ A. Ishizaka and Y. Shiraki, J. Electrochem. Soc. 133, 666 (1986).

${ }^{15}$ R. J. Hamers, R. M. Tromp, and J. E. Demuth, Phys. Rev. Lett. 56, 1972 (1986).

${ }^{16}$ Emission from $\mathrm{Si}-\mathrm{H}$ bonds does not appear in or near normal emission: K. Hricovini et al., Phys. Rev. Lett. 70, 1992 (1993). 